\title{
$\mathrm{ICONO} 14$
}

\section{Neuromarketing: concepto, evolución histórica y retos}

\section{Neuromarketing: concept, historical evolution and challenges}

\section{Neuromarketing: conceito, evolução histórica e desafíos}

\author{
Coral Cenizo ${ }^{1}$ iD \\ ${ }^{1}$ Investigadora (Universidad San Pablo CEU), España
}

Recibido: 29/06/2021; Revisado: 14/07/2021; Aceptado: 07/10/2021; Publicado: 01/01/2022

Para citar este artículo: Cenizo, C. (2022). Neuromarketing: concepto, evolución histórica y retos. Icono 14, 20(1). https://doi.org/10.7195/ri14.v20i1.1784

\section{Resumen}

En un mundo cada vez más competitivo, muchos expertos y teóricos siguen buscando la respuesta al Santo Grial del marketing: ¿qué lleva a los consumidores a elegir un producto en lugar de otro? ¿Qué factores influyen en la percepción que tiene un consumidor de una marca? Muchos creen que la clave está en el neuromarketing. Sin embargo, esta disciplina se enfrenta hoy a un profundo desconocimiento, tanto en la comunidad científica como empresarial, que está originando incertidumbre sobre las posibilidades reales que ofrece. Este artículo pretende arrojar más luz sobre esta materia, analizando para ello su evolución histórica — desde sus orígenes hasta la actualidad-, así como su conceptualización y los retos a los que se enfrenta, mediante una revisión exhaustiva de la literatura científica publicada hasta el momento. Los resultados ofrecen un completo estado de la cuestión, esencial para entender el significado real del término neuromarketing, la situación de inestabilidad en la que se encuentra la disciplina, las limitaciones que están constriñendo su desarrollo y las carencias investigadoras. El artículo 
constituye una piedra angular sobre la que sustentar futuras investigaciones o aplicaciones prácticas del neuromarketing.

Palabras clave: publicidad; toma de decisiones; consumidor; neuromarketing; neurociencias; investigación de mercados

\begin{abstract}
In an increasingly competitive world, many experts are searching for the answer to the Holy Grail of marketing: what drives consumers to choose one product over another? What factors influence a consumer's perception of a brand? Many believe that the key is neuromarketing. However, this discipline faces a deep lack of knowledge, both in the scientific and business communities, which is causing uncertainty about the real possibilities it offers. This article aims to shed more light on this matter, analyzing its history - from its origins to the present-, as well as its conceptualization and the challenges it faces, through an exhaustive review of scientific literature. The results offer a complete state of the art, essential to understand the real meaning of the term neuromarketing, the situation of instability in which the discipline finds itself, the limitations that are constraining its development and the research deficiencies to date. This article constitutes a cornerstone to support future research or practical applications of neuromarketing.
\end{abstract}

Keywords: advertising; decision making; consumer; neuromarketing; neuroscience; market research

\title{
Resumo
}

Em um mundo cada vez mais competitivo, muitos especialistas e teóricos continuam em busca da resposta para o Santo Graal do marketing: o que leva os consumidores a escolher um produto em vez de outro? Que fatores influenciam a percepção do consumidor sobre uma marca? Muitos acreditam que a chave está no neuromarketing. No entanto, essa disciplina hoje enfrenta um profundo desconhecimento, tanto na comunidade científica como empresarial, o que vem gerando incertezas sobre as reais possibilidades que oferece. Tendo em conta o pequeno número de artigos científicos publicados em espanhol, este artigo pretende lançar mais luz sobre esse assunto, analisando a sua evolução histórica —desde as origens até ao presente-, bem como a sua conceituação e os desafios que enfrenta, por meio de uma revisão exaustiva da literatura científica relacionada ao neuromarketing. Os resultados oferecem um estado da arte, essencial para compreender o real significado do termo neuromarketing, a situação de instabilidade em que a disciplina se encontra, as limitações que condicionam o seu desenvolvimento e as deficiências da investigação até à data. $\mathrm{O}$ artigo constitui uma pedra angular sobre a qual apoiar pesquisas futuras ou aplicações práticas do neuromarketing.

Palavras-chave: publicidade; tomada de decisão; consumidor; neuromarketing; neurociência; pesquisa de mercado 


\section{Introducción}

Durante años, los modelos económicos tradicionales defendieron que eran las motivaciones racionales de los consumidores, marcadas por el cumplimiento de sus propios intereses, las que marcaban los procesos de compra (Thompson, 2013). Aunque esta afirmación no deja de ser cierta, numerosos estudios (Dooley, 2011, y Ramsoy, 2014, por citar dos ejemplos) han puesto en duda que las decisiones de compra se guíen únicamente por la racionalidad; de hecho, algunos expertos defienden que nuestras decisiones de compra son más emocionales de lo que creemos y que la concepción de que el ser humano es una máquina cognitiva movida por decisiones racionales está desfasada (Lindstrom, 2010; Shiv y Fedorikhin, 1999; Sinek, 2009; Hazeldine, 2014).

Precisamente por esto, en el marketing actual ya no solo se busca que el producto o servicio posea una ventaja competitiva racional, sino que el consumidor tenga a su vez palancas emocionales y una experiencia de compra lo suficientemente atractiva como para influir en su decisión de compra, más allá de sus límites objetivos (Levy y Weitz, 2009). El entendimiento, por tanto, del inconsciente de los consumidores es esencial hoy en día.

En todo este contexto, el neuromarketing ha aparecido como una posible solución a este enigma hasta el punto de que algunos autores hablan de que su estudio y desarrollo marcará un antes y un después en el sector del marketing (Perrachione y Perrachione, 2008).

Sin embargo, y a pesar de su potencial para evaluar las reacciones subliminales del consumidor ante marcas, productos o materiales de marketing y determinar qué elementos influyen más en la toma de decisiones de compra, existe un profundo desconocimiento sobre esta disciplina. Esto está generando a su vez una visión limitada de la cuestión, malentendidos y una fama inmerecidamente errónea que está propagando imágenes muy distorsionadas sobre lo que es realmente el neuromarketing y las posibilidades que ofrece (Dooley, 2015; Samuel y Prasanth, 2012).

\section{Método}

El presente artículo se basa en tres objetivos investigadores principales; en primer lugar, indagar en el término neuromarketing para conocer su esencia y ámbito. Existe en la actualidad una desvirtuación de la palabra neuromarketing, hasta el punto de que se usa de forma indiscriminada y errónea por parte de los medios de comunicación y los ciudadanos en general (Monge y Fernández Guerra, 2012). Se hace preciso, por tanto, concretar una definición completa y correcta a fin de corregir estas desviaciones. 
En segundo lugar, se pretende estudiar la evolución histórica del neuromarketing para entender de qué forma ha ido cambiando la percepción de la disciplina, su existencia dentro del ámbito científico y profesional, así como las polémicas surgidas a su alrededor, desde su nacimiento hasta la actualidad. Con este análisis histórico se busca crear un marco general que permita entender de qué forma esta disciplina ha llegado a la situación actual en la que se encuentra.

En tercer y último lugar, pretendemos analizar los principales retos a los que se enfrenta hoy en día el neuromarketing; la comprensión de estos desafíos es esencial para detectar vías de mejora y también para entender qué aspectos están limitando el desarrollo del neuromarketing y su aplicación generalizada en el ámbito profesional e investigador.

Para dar respuesta a estos tres objetivos se ha realizado una exhaustiva revisión de la literatura científica relacionada con el neuromarketing, desde las investigaciones pioneras publicadas en los primeros años de los 2000 hasta las más recientes, que analizan las aplicaciones más recientes del neuromarketing. En total, se han estudiado 67 fuentes -entre revistas científicas indexadas y obras de referencia- que han obtenido una repercusión destacada en el ámbito científico por su calidad o resultados.

Sobre este corpus se ha llevado a cabo un análisis teórico de tipo cualitativo, utilizando para ello un enfoque descriptivo que no pretende valorar el objeto de estudio sino analizar su naturaleza de la forma más objetiva posible a fin de construir una base teórica lo más aséptica posible.

\section{Desarrollo}

\subsection{El concepto de neuromarketing}

El término neuromarketing surgió en 2002 cuando el profesor Ale Smidts publicó su artículo "Kijken in het brein" ("Mirando en el cerebro"); sin embargo, cuando el artículo se tradujo al inglés, el título de este se adaptó a "Looking into neuromarketing", introduciendo así el término neuromarketing por primera vez en el sector (Smidts, 2002). No obstante, aunque se atribuye a Smidts la acuñación del término neuromarketing (Orzan, Zara y Purcarea, 2012), el origen de la disciplina se remonta a 1999, año en el que se realizó el primer estudio fMRI (functional magnetic resonance imaging) con fines de marketing a cargo del profesor Gerry Zaltman (Roeduck, 2011).

Tal vez debido a su complejidad, se han acuñado numerosas definiciones del término neuromarketing. Smidts definió el concepto como el estudio de los mecanismos y procesos 
cerebrales para comprender los patrones y pautas de comportamiento del consumidor con el objeto de mejorar las estrategias de marketing (2002).

Un planteamiento similar han usado Lee, Broderick y Chamberlain quienes entienden que el neuromarketing supone la aplicación de métodos neurocientíficos a fin de analizar y comprender el comportamiento humano frente a estímulos de marketing; en otras palabras: con el neuromarketing se busca entender la forma de pensar de los consumidores y las razones que les empujan a elegir un producto o marca frente a otra aplicando para ello método neurocientíficos (2007). Con esta definición, los autores sentaron ya unas bases importantes pues ampliaron el alcance del neuromarketing, abarcando aspectos que van más allá del estudio del comportamiento del consumidor.

Más precisa aun es la definición aportada por Dooley para quien el neuromarketing es un área nueva dentro de la investigación de marketing que estudia las respuestas cognitivas y emocionales de los consumidores ante diferentes estímulos de marketing (2010).

Lo que parece claro es que el neuromarketing es un campo interdisciplinario que combina elementos de la neurociencia y la psicología con teorías propias del comportamiento de consumidor a fin de explicar qué mueve al consumidor a realizar su decisión de compra final (Marichamy y Sathiyavathi, 2014). En un principio, surgió como parte de la neuroeconomía, de ahí que se considere un campo situado entre las neurociencias y la economía cuyo objetivo es explicar el proceso de toma de decisiones mediante el desarrollo de modelos y sistemas neuronales (Pop et al., 2014; Egidi, 2008).

Existen diversas técnicas dentro del neuromarketing; estas pueden dividirse entre aquellas que registran la actividad dentro del cerebro y aquellas que registran la actividad fuera del cerebro. La primera técnica del primer grupo es la Resonancia Magnética Funcional (fMRI) que mide, mediante magnetismo, cambios en la oxigenación de la sangre, a su vez relacionados con la actividad del cerebro; de esta forma, la fMRI ofrece imágenes del cerebro mientras éste está funcionando para analizar las respuestas neuronales frente a estímulos. La segunda técnica es la electroencefalografía o EEG que analiza, mediante unos electrodos colocados en el cuero cabelludo, las zonas del cerebro en las que se está produciendo mayor actividad; a diferencia de la fMRI, el coste de la EEG es más reducido lo que ha hecho que sea una de las técnicas más utilizadas dentro del neuromarketing. La tercera técnica es la magnetoencefalografía o MEG; similar a la EEG, la MEG mide los campos magnéticos que se producen con la actividad neuronal; la MEG ofrece mejor calidad y resolución temporal que la EEG pero el alto coste del equipamiento necesario hace que su uso sea muy reducido.

En el segundo grupo encontramos la electromiografía o EMG, que consiste en la aplicación de electrodos de bajo voltaje en los músculos para estudiar la actividad eléctrica que estos generan cuando se producen respuestas emocionales involuntarias; normalmente la EMG se utiliza en los músculos faciales para controlar expresiones muy breves e inapreciables a simple vista. Otra técnica es la Facial Action Coding System (FACS) 
que mide, mediante la observación de las expresiones faciales, la reacción muscular ante determinados estímulos; esta técnica empezó a utilizar en la década de los setenta a través de los experimentos desarrollados por Paul Ekman; en la actualidad se desarrolla a través de softwares específicos (Azcarate et al., 2005). La tercera técnica de este grupo es el seguimiento ocular o eye-tracking;en este caso se analiza hacia dónde mira el sujeto, algo especialmente útil si existen múltiples estímulos o si se necesita información contextual.

A pesar de la diversidad de técnicas vigentes, todas ellas trabajan en una misma dirección, esto es, estudiar mediante la actividad corporal las respuestas cognitivas, sensoriomotoras y afectivas de los consumidores ante estímulos de marketing concretos (Ohme et al., 2009) y determinar si ciertos cambios en estos estímulos provocan a su vez modificaciones en las señales corporales (Kenning y Plassmann, 2008; Riccio et al., 2015; Rodrigues, 2011).

Los partidarios defienden que el neuromarketing aporta información imposible de obtener mediante los procesos habituales de investigación de marketing, tales como entrevistas, cuestionarios o focus groups (Ariely y Berns, 2010); esto se debe a que las personas pueden no decir la verdad cuando son preguntadas de forma explícita algo que, sin embargo, el neuromarketing pasa por alto (Calvert y Brammer, 2012). Para otros autores, intentar que los consumidores expresen de una manera racional sus emociones acerca de un producto o material de marketing no puede considerarse una fuente de información válida; la parcialidad que indudablemente se deriva de este procedimiento hace que su eficiencia sea cuestionable (Oliver, 2016). De hecho, es esta la principal diferencia entre el neuromarketing y las técnicas de investigación tradicionales: el neuromarketing ofrece una ventana abierta al cerebro de un consumidor que deja al descubierto detalles por lo general encubiertos que pueden suponer la diferencia entre una campaña de marketing exitosa y otra fracasada (Colaffero y Crescitelli, 2014; Marichamy y Sathiyavathi, 2014; Mucha, 2005).

El valor potencial del neuromarketing es incalculable; tal y como señalaron Arthmann y Li, no hay duda de que los responsables de marketing buscan de manera incansable descubrir los secretos del subconsciente de los consumidores (2017); el coste de algunas campañas publicitarias o el desarrollo de un producto puede superar en muchos casos varios millones de euros; en este sentido, el uso de técnicas de neuromarketing parece razonable si con ello se reduce el riesgo en la inversión de marketing.

\subsection{Evolución histórica del neuromarketing}

A pesar de su consolidación en 2002, el ámbito del neuromarketing evolucionó con cierta lentitud a partir de entonces. No fue hasta 2004 cuando empezaron a surgir artículos académicos como el estudio Coca-Cola vs Pepsi, considerado un referente en el sector incluso en la actualidad. En este estudio, se pidió a un grupo de sujetos que bebieran 
Coca-Cola y Pepsi al tiempo que sus cerebros eran monitoreados mediante resonancia magnética funcional (fMRI). El experimento arrojó resultados claros: se detectó respuesta neuronal consistente en la corteza prefrontal ventromedial que se correlacionó con las preferencias de los sujetos hacia cada una de estas bebidas. También se demostró que el conocimiento de la marca influía en las preferencias de comportamiento expresadas y en las respuestas cerebrales medidas (McClure et al., 2004).

Poco después, nacieron las dos primeras consultoras que ofrecían servicios de investigación y consultoría utilizando para ello técnicas de neuromarketing: BrightHouse y SalesBrain; con esto, se asentó la adopción de tecnologías asociadas a las neurociencias dentro del ámbito empresarial y del marketing (Fisher et al., 2010; Alwitt, 1985).

Los siguientes años estuvieron marcados por las posturas enfrentadas. Por un lado, se generó un gran optimismo sobre las posibilidades que abría el neuromarketing; muestra de ello son las palabras de Pop, Radomir, loana y Maria quienes afirmaron que la única forma de medir de forma objetiva los pensamientos y sentimientos de los consumidores era a través de las neurociencias (2009). Este optimismo también coincidió con el abaratamiento de las técnicas de neuromarketing que hizo más asequible su uso y testeo.

Fugate, por ejemplo, llegó a presentar una lista con las áreas en las que el neuromarketing era especialmente útil frente a las técnicas tradicionales de investigación de marketing, tales como la efectividad publicitaria, la selección de logos, el atractivo de determinados productos, etc. (Fugate, 2008).

No obstante, ya por aquel entonces surgieron también críticas; algunos expertos apuntaron a que el neuromarketing era poco ético, superando los límites incluso de la legalidad (Lee et al., 2007). Otros hablaron de los altos costes asociados a las diferentes técnicas que no compensaban los resultados arrojados (Hubert y Kenning, 2008) e incluso de la influencia negativa que podía causar en la sociedad pues sentaba un precedente que podía derivar en la manipulación y control de los individuos por parte de las marcas (Murphy et al., 2008).

También hubo quienes mantuvieron una posición de escepticismo, argumentando que el neuromarketing era tan solo una moda pasajera que no iba a ofrecer ningún Santo Grial que permitiera descubrir el botón misterioso que impulsaba a un consumidor a comprar (Lewis y Bridger, 2005).

Dejando al margen la controversia, el neuromarketing empezó a alcanzar popularidad en los años siguientes, en parte por la esperanza que se tenía de que las diferentes técnicas fueran cada vez más baratas y rápidas frente a otros métodos de investigación, y también por el hecho de que el neuromarketing aportara información que no se podía obtener de otra forma (Ariely y Berns, 2010). Empezó a verse el neuromarketing como un campo exento de magia, con sus propias limitaciones, pero que ofrecía información de valor para 
entender las complejidades de las decisiones de compra de los consumidores (Yoon et al., 2012).

Así, entre 2010 y 2015 se publicaron numerosos artículos en los que se analizaron las ventajas y desventajas de las diferentes técnicas asociadas al neuromarketing, lo que hizo que el campo empezara a cobrar más fuerza como herramienta investigadora fiable (Bercea, 2012).

Pero el neuromarketing no solo captó la atención del mundo académico e investigador sino también del empresarial, lo que hizo que aumentara el número de empresas y consultoras dedicadas y especializadas en este campo (Plassmann et al., 2012). También el número de búsquedas del término en Internet se incrementó: de las 800.000 búsquedas en 2008 se pasó al casi millón y medio en 2021 y de ahí a los tres millones en 2018 (Hubert y Kenning, 2008).

Este auge quedó consolidado con la fundación en 2012 de la Neuromarketing Business and Science Association (NMBSA), cuyo cometido, más allá de promocionar el neuromarketing a nivel mundial, fue asentar una base metodológica para este campo y favorecer así su aceptación en la comunidad científica.

Pero a pesar de la creciente popularidad, el neuromarketing siguió enfrentándose a duras críticas; se puso en duda, por ejemplo, la eficacia de los métodos del neuromarketing y se descartó que pudiera sustituir a las técnicas de investigación tradicionales debido a que las condiciones de los experimentos y de las metodologías limitaba su generalización (Ariely y Berns, 2010; Solnais et al., 2013).

Pero a pesar de la cautela que mostraban los científicos y las escasas investigaciones empíricas, empresas especializadas en marketing como Nielsen o Millward Brown crearon departamentos especializados en neuromarketing para aplicar las últimas técnicas en las estrategias de marketing de sus clientes (Plassmann et al., 2015).

Fue a partir de 2015 cuando se presentaron los primeros resultados relevantes sobre la materia; en su artículo "Individual Differences in Marketing Placebo Effects", Plassmann y Weber investigaron el efecto placebo (MPE) en el ámbito del marketing; concretamente, en esta investigación se analizó las diferencias en las respuestas al placebo que presentaban los individuos, utilizando para ello técnicas basadas en la actividad cerebral (2015).

Otro artículo interesante fue el presentado por Chen, Nelson y Hsu; combinando técnicas de machine learning con neuroimagen funcional se llegó a la conclusión de que, a diferencia de construirse a través de procesos reflexivos, la personalidad de una marca existe dentro de la mente de los consumidores; así, los investigadores pudieron predecir en qué marca pensaban los individuos que formaban parte del estudio basándose únicamente en la relación entre las asociaciones de personalidad de la marca y la actividad cerebral (2015). 
Pero a pesar de los mencionados avances, en los años sucesivos hasta la actualidad, han sido pocos los estudios científicos realizados sobre la materia; la mayoría de las investigaciones adolecen de fundamento empírico y aportan, por el contrario, revisiones de resultados pasados, planteamientos personales o experimentos que, pese a su potencial, no están logrando progresos reales o extrapolables, a excepción de despertar el interés por la investigación futura (Lin et al., 2018; Krampe et al., 2018).

Aun así, en los últimos años se ha detectado un interés creciente por aspectos relacionados con el neuromarketing, como su dimensión ética; muestra de ello es el artículo firmado por Stanton, Sinnott-Armstrong y Huettel en el que se detallan los riesgos éticos derivados de las técnicas y usos del neuromarketing y también diferentes soluciones con los que mitigarlos (2017). Similar planteamiento mantienen Hensel, lorga, Wolter y Znanewitz quienes, a través de una serie de entrevistas a profesionales del neuromarketing, constataron la importancia de mantener y respetar los aspectos éticos del neuromarketing a fin de asegurar tanto la calidad de los resultados como la protección de los participantes. Los autores también aportaron cinco medidas éticas adicionales para garantizar la transparencia y objetividad del neuromarketing (2017).

También en los últimos años se aprecia un cambio de perspectiva en la concepción del neuromarketing; frente a la autonomía e independencia de la materia defendida en los primeros años, en la actualidad existe una postura más templada que considera el neuromarketing como una técnica de investigación complementaria, no sustitutiva, de las metodologías tradicionales para entender la toma de decisión de los consumidores (Stanton y al., 2017; Hsu, 2017). Buena muestra de este papel suplementario del neuromarketing lo encontramos en la investigación planteada por Nave, Nadler, Dubois, Zava, Camerer y Plassmann en la que el análisis de la actividad cerebral reafirmó los resultados obtenidos mediante la correlación entre los niveles de testosterona y las preferencias de los consumidores por las marcas de lujo (2018).

Tampoco hay que desdeñar los avances recientes en la conceptualización del neuromarketing; por ejemplo, Knutson y Genevsky plantearon en 2018 un nuevo concepto relacionado con la materia: neuroforecasting; con este término, los autores definieron la capacidad de prever las futuras decisiones de compra de los consumidores utilizando para ello imágenes cerebrales. Knutson y Genevsky planteaban este concepto tanto para sujetos individuales como para grupos lo que permitiría predecir comportamientos agregados. No obstante, los autores también invitaban a la cautela al reconocer que el neuroforecasting es una técnica aún por evolucionar y estudiar (2018).

Así mismo reciente es la aplicación del neuromarketing al ámbito digital; en este sentido, destacan los estudios planteados por Arthmann y Li y Constantinescu, Orindaru, Pachitanu, Rosca, Caescu y Orzan. En el primer caso, los investigadores se centraron en la vinculación entre neuromarketing e Internet, así como en los beneficios derivados de dicha unión; concluyeron que el neuromarketing tiene puede influir en la asociación de la marca y la lealtad del consumidor de forma proactiva, ya que mide el comportamiento de los clientes 
en tiempo real, tanto sus respuestas verbales como no verbales hacia nuevos productos, precios y promociones publicitarias (2017).

En el caso de la segunda investigación se analizó el uso del neuromarketing en las redes sociales, considerando los propósitos de la empresa y los beneficios del consumidor. Para ello, se realizaron dos investigaciones a partir de las cuales los autores crearon un modelo donde estos propósitos se combinaban con los correspondientes beneficios, mostrando el grado de aceptabilidad de cuatro grandes aplicaciones del neuromarketing para lograr un crecimiento empresarial sostenible. Aun así, los resultados demostraron que no era sencillo aplicar técnicas del neuromarketing a las redes sociales debido a dos motivos: en primer lugar, la alta inversión que supone para las empresas la compra del equipamiento necesario para hacer las mediciones, $y$, en segundo lugar, la falta de disposición de los individuos para formar parte de este tipo de investigaciones (2019).

Menos dramática es la evolución en las críticas hacia el neuromarketing; si bien es cierto que se han moderado con los años las alusiones al carácter negativamente distópico del neuromarketing, se mantienen las dudas sobre su eficacia real; tal es el caso, de Hsu quien apela a la complejidad del sistema nervioso para cuestionar la correspondencia entre regiones específicas del cerebro y los estados mentales, especialmente aquellos valiosos para el marketing como la lealtad o el amor. Hsu va más allá al reconocer que es un completo error buscar una única causa en la efectividad de las acciones de marketing pues el éxito de estas por lo general deviene de múltiples factores; pone de ejemplo los anuncios cuyo éxito, según Hsu, suele proceder de una combinación de riqueza emocional, visualización placentera y carácter llamativo (2017).

Otros autores como Lim critican el bagaje investigador previo, argumentando que los estudios precedentes sobre la materia ofrecen una guía muy limitada sobre cómo debe realizarse una investigación sólida basada en el neuromarketing; dado que solo analizan aspectos básicos de los diferentes métodos neurocientíficos, disuaden más que ayudan al desarrollo de investigaciones de marketing basadas en el neuromarkerting (2018).

Una posición similar mantienen Lee, Chamberlain y Brandes quienes reconocen que la enorme fragmentación de las investigaciones previas, la falta de metodología de calidad orientada al usuario y la ausencia de guía que permita distinguir una correcta investigación de neuromarketing están limitando el crecimiento potencial de esta área (2018).

\subsection{Retos del neuromarketing}

El neuromarketing es un campo en crecimiento, con una gran variedad de facetas que cada año despiertan más interés en la comunidad científica (Lee y et al., 2018); no obstante, el área se enfrenta a importantes retos, que recogemos a continuación. 


\subsubsection{Costes}

El neuromarketing no es barato; muy al contrario, el uso de técnicas vinculadas a las neurociencias supone un alto coste, tanto por la compra de materiales como por la puesta en marcha de los experimentos. Esto supone, según Hilderbrand, el principal escollo de este campo por su carácter limitador (2016); como marco general, el coste de estudios de eye-tracking y EEG se sitúa entre los 5.000-15.000 dólares por anuncio. En el caso de que un anunciante quiera testar más de un anuncio, el coste de un pretest para un grupo de anuncios ronda los 20.000-35.000 dólares. Incluso los equipos más baratos -la mayoría de ellos asociados a la EEG - implican un gasto de 10.000 (Ariely y Berns, 2010).

Pero los gastos no solo devienen del equipamiento y ejecución del experimento; también hay que considerar otros factores que pueden aumentar el coste como, por ejemplo, la selección de individuos con rasgos especiales, el tamaño de la muestra, mayor rapidez en la obtención de resultados o la inclusión de múltiples estímulos.

Los altos costes de técnicas como fMRI o la MEG ha hecho que las consultoras se decanten por opciones más económicas como es el caso de la EEG que, sin embargo, plantean algunas limitaciones como la resolución espacial limitada o la ausencia de datos procedente de las capas internas del cerebro. Otros expertos ponen en duda la eficacia del neuromarketing desde el punto de vista del ROI, si bien es cierto que hasta la fecha no se ha realizado ningún estudio concreto al respecto (Monge y Fernández Guerra, 2012).

\subsubsection{Tamaño de la muestra}

El segundo aspecto más controvertido es el tamaño de las muestras. Como hemos mencionado antes, el número de individuos que forman parte del experimento influye considerablemente en el precio de los experimentos. Esto ha hecho que muchas consultoras especializadas en neuromarketing reduzcan los grupos de muestreo para ajustar al máximo los costes de sus servicios. La mayoría de estas empresas defienden que la calidad de los resultados no es proporcional con el número de individuos que forman parte del experimento; de hecho, según Sands Research una muestra de entre 30 y 40 sujetos arroja apenas un 1\% de error con la tecnología EEG (Sands, 2009).

El contraste con las metodologías empíricas tradicionales es clara: por lo general, los estudios de mercado requieren un número considerable de respuestas para considerarse válidas, normalmente superando el centenar.

Esta diferencia ha hecho que muchos expertos cuestionen la extrapolación de datos procedentes del neuromarketing. Henrich, por ejemplo, afirma que es imposible obtener resultados concluyentes sin los tamaños muestrales adecuados; el autor critica además la 
generalización de los resultados a la muestra poblacional total tomando como referencia un subgrupo muy concreto, algo muy habitual en el neuromarketing (2010).

Tampoco existe un consenso claro entre las consultoras especializadas; mientras algunas, como Sands defienden a ultranza la eficacia de los muestreos de 30 a 40 personas, otras, como EmSense, cuestionan estos números y mantienen que son necesarios muestras de mayor tamaño, similares a las utilizadas en otras técnicas investigadoras, para llegar a lo que ellos denominan «neuromarketing cuantitativo» (Monge y Fernández Guerra, 2012).

\subsubsection{Regulación ética}

La regulación ética del neuromarketing es uno de los principales retos a los que se enfrenta esta disciplina. El rechazo que provocan las técnicas del neuromarketing en los individuos es claro; según Lindstrom, el neuromarketing provoca cierta incomodidad en los individuos; esto se debe al miedo general que existe al dar acceso al cerebro, considerada una intrusión mayor con potencial para revelar nuestros pensamientos y sentimientos más profundos. Igual que un siniestro Peeping Tom, las neurociencias son más temidas que queridas, en parte también por el desconocimiento que existe alrededor de sus procedimientos (2008).

En efecto, el neuromarketing no está exento de dilemas éticos, muchos de los cuales quedaron recogidos por Murphy, Illes y Reiner en su artículo "Neuroethics of neuromarketing" (2008). Por ejemplo, no existe un filtrado en la información que obtienen las diferentes técnicas de neuromarketing de los individuos, de forma que estos no pueden elegir qué información compartir y cuál no. Cierto es, sin embargo, que, hoy en día la información que puede extraer el neuromarketing es muy limitada (Tovino, 2005).

Otro aspecto polémico es la ausencia de regulación específica; a diferencia de las investigaciones clínicas, el neuromarketing no cuenta con unas normas reguladas por una entidad independiente que garanticen su correcta aplicación (Illes et al., 2006); así, por ejemplo, no existen procedimientos de actuación en los casos en los que se detectan problemas de salud -que ocurre aproximadamente en el 1\% de los casos-o para la protección de individuos con vulnerabilidades neurológica.

En efecto, el desarrollo actual de estas técnicas es bastante limitado, pero queda en el aire que su posterior evolución pueda derivar en procesos de manipulación subliminal o incluso de control mental que lleve a los individuos a actuar contra su voluntad. Este es un miedo que tradicionalmente siempre ha existido en la disciplina y que han planteado de forma reiterada diversos autores (Murphy et al., 2008). 


\subsubsection{Ausencia de estándares}

La ausencia de unos estándares comunes para la disciplina hace que cada consultora aplique unas metodologías propias para llevar a cabo sus experimentos, siguiendo sus propios criterios de calidad -tanto en la recogida de datos como en la aplicación de tecnologías-, por lo que el análisis de los resultados es diferente según la consultora. Esta diversidad total de metodologías hace que los resultados sean difícilmente comparables entre sí (Monge y Fernández Guerra, 2012). Así, resulta imposible el trabajo paralelo con dos o más consultoras para la posterior comparación de resultados, una práctica, por otro lado, habitual en otros campos.

La juventud de la disciplina y la competencia del mercado parecen ser las principales causas de esta diversidad; no hay que olvidar que la guerra de precios imperante entre las empresas especializadas en el sector obliga a imponer un secretismo en las metodologías que hace imposible la creación de unos estándares conjuntos (Monge y Fernández Guerra, 2012).

Existe, no obstante, una voluntad por parte del sector para establecer unos estándares válidos; el caso más claro es la iniciativa Neurostandards Collaboration, liderada por la Advertising Research Foundation (ARF); esta iniciativa reunió a ocho grandes consultoras -Gallup y Robinson, Innerscope, LABoratory, Mindlab International, NeuroCompass, Neuro-Insight, Sands Research y Sensory Logic- a fin de intercambiar conocimientos y fundamentar unos estándares válidos para todos. La ausencia de dos de las consultoras líderes en el mercado, Neurofocus y EmSense, así como los resultados poco prometedores de la primera fase - publicados en 2011- condicionó, sin embargo, las expectativas iniciales con las que nació Neurostandards Collaboration.

\section{Conclusión}

Con el presente estudio pretendíamos dar respuesta a tres preguntas de investigación. En primer lugar, concretar el concepto de neuromarketing de la forma más completa posible; en este sentido, se ha podido definir, mediante las aportaciones de diferentes autores, el concepto de neuromarketing, así como sus ámbitos y técnicas de actuación más habituales. También ha quedado demostrado que la conceptualización del neuromarketing trasciende los límites terminológicos; la acuñación del término fue la que dotó a la disciplina de entidad y la que asentó su presencia en el ámbito científico y profesional pues es a partir de entonces cuando se aprecia un mayor interés por la cuestión en todos los ámbitos. También interesante es el hecho de que a pesar de que el nacimiento del vocablo se fije en 2002, ya existían antes de esa fecha investigaciones precedentes que utilizaron técnicas propias de las neurociencias con fines de marketing - como la conducida por Gerry Zaltman-, a pesar de la ausencia del término apropiado 
para definirlo; por lo tanto, su acuñación fue fruto de la necesidad creada en el ámbito investigador.

El segundo objetivo de la investigación era definir el marco histórico del neuromarketing, a fin de entender la compleja situación a la que se enfrenta esta disciplina, así como sus limitaciones.

En este sentido, el análisis de la evolución histórica del neuromarketing nos ha permitido comprender mejor el camino de altibajos por el que ha atravesado esta disciplina, marcado en su mayoría por las posturas enfrentadas, las dudas y las incertidumbres. Bajo este marco es fácil entender la situación de inestabilidad a la que se enfrenta hoy esta disciplina, todavía en búsqueda de una identidad propia y sometida a continuos debates.

Así mismo, nos ha permitido detectar dos cuestiones de suma importancia.

En primer lugar, desde su nacimiento, se ha atribuido al neuromarketing capacidades que, al menos por el momento, superan la realidad; muy lejos de ser la clave absoluta para desentrañar los secretos de las decisiones de compra, el neuromarketing ha demostrado ser una metodología complementaria, de alcance limitado, que está muy lejos aún de los objetivos que pretendía resolver. Al mismo tiempo, el neuromarketing ofrece, hasta la fecha, información similar a la de otras técnicas, muy lejos de las posibilidades que se le ha asignado históricamente, tales como manipulación de los individuos, cambios de las estructuras cerebrales, entre otras (Levy, 2009). Si el neuromarketing pudiera evolucionar o no hasta alcanzar estas cotas, es difícil de prever con seguridad. Es necesaria cierta cautela a la hora de valorar las posibilidades futuras para no caer en puntos de vista exageradamente optimistas ni pesimistas.

En segundo lugar, es obvio que el neuromarketing adolece de trayectoria investigadora; esto puede deberse a su relativa juventud, que aún no ha dado lugar a un bagaje ni una metodología sólida. Sin embargo, esta ausencia está perjudicando, más que beneficiando, a la disciplina; como ya adelantó Dooley, la falta de trabajos científicos de peso está generando una falta de comprensión y entendimiento dentro de la industria (2015). Así, se hace necesario aumentar las investigaciones sobre el neuromarketing para conocer el potencial real de esta disciplina en áreas como el marketing o el desarrollo de productos, determinar hasta qué punto se puede extrapolar estas técnicas a la práctica de una forma eficiente y paliar la falta de conocimiento que existe sobre la cuestión (Butler, 2008).

Con respecto al tercer objetivo investigador, con el que pretendíamos precisar los retos a los que se enfrenta el neuromarketing en la actualidad, es innegable que el interés por el neuromarkerting está creciendo; son cada vez más las empresas y expertos que ven en esta relativamente nueva metodología una puerta hacia el futuro que permita desarrollar y crear acciones de marketing más efectivas y seguras a nivel económico. Sin embargo, el neuromarketing se enfrenta, tal y como hemos visto en esta investigación, 
a importantes desafíos que están lastrando su desarrollo y que pueden condicionar su futuro más próximo.

Los altos costes que implica la puesta en marcha de las diferentes técnicas del neuromarketing, la discrepancia que existe en torno al tamaño óptimo de las muestras, la ausencia total de estándares que hace imposible la comparación de resultados y, sobre todo, los dilemas éticos, son cuatro importantes escollos que ponen en tela de juicio la eficacia y crecimiento del neuromarketing.

Pero, a pesar de las limitaciones actuales, las posibilidades del neuromarketing son enormes; los avances que se han hecho hasta la hecha ofrecen el suficiente optimismo como para confiar en las capacidades futuras de esta disciplina. Los próximos años serán claves para fundamentar las bases de este campo, corregir las deficiencias actuales, crear estándares metodológicos y éticos y reducir los costes para hacer de esta disciplina una herramienta más al servicio de la investigación de mercado.

\section{Referencias}

Alwitt, L. (1985). EEG activity reflects the content of commercials. En Alwitt, L., Psychological processes and advertising effects: theory, research, and applications (pp. 209-219). Hillsdale, NJ: Lawrence Erlbaum.

Ariely, D., \& Berns, G. S. (2010). Neuromarketing: the hope and hype of neuroimaging in business. Nature reviews Neuroscience, 11(4), 284-292. https://doi.org/10.1038/nrn2795.

Arthmann, C., \& Li, I.P. (2017). Neuromarketing - The Art and Science of Marketing and Neurosciences Enabled by loT Technologies. IIC Journal of Innovation, 1-10.

Azcarate, A., Sande, K.V., \& Valenti, R.G. (2005). Automatic facial emotion recognition. https://bit.ly/3 goKE5p

Bercea, M. D. (2012). Anatomy of methodologies for measuring consumer behavior in neuromarketing research. https://bit.ly/355BhCe

Butler, M. (2008). Neuromarketing and the perception of knowledge. Journal of Consumer Behavior, 7, 415-419. https://doi.org/10.1002/cb.260.

Calvert, G.A., \& Brammer, M.J. (2012). Predicting consumer behavior. IEE Pulse Magazine, 3(3), 38-41. https://doi.org/10.1109/MPUL.2012.2189167.

Chen, Y.P., Nelson, L.D., \& Hsu, M. (2015). From "Where" to "What": Distributed Representations of Brand Associations in the Human Brain. Journal of Marketing Research, 52(4), 453-466. https:/ /doi.org/10.1509/jmr.14.0606.

Colaferro, C.A., \& Crescitelli, E. (2014). The Contribution of Neuromarketing to the Study of Consumer Behavior. Brazilian Business Review, 11(3), 123-143. https://doi.org/10.15728/bbr.2 014.11.3.6.

Constantinescu, M., Orindaru, A., Pachitanu, A., Rosca, L., Caescu, S.C., \& Orzan, M. C. (2019). Attitude Evaluation on Using the Neuromarketing Approach in Social Media: Matching Company's Purposes and Consumer's Benefits for Sustainable Business Growth. Sustainability, 11(24), 7094. https://doi.org/10.3390/su11247094. 
Dooley, R. (2010). Baby pictures do really grab our attention. https://bit.ly/3g4c2Xo.

Dooley, R. (2011). Brainfluence: 100 ways to persuade and convince consumers with Neuromarketing. USA: Wiley.

Dooley, R. (24 de febrero de 2015). Neuromarketing: Pseudoscience no more. https://bit.ly/3g4n7ba.

Egidi, G., Nusbaum, H.C., \& Cacioppo, J.T. (2008). Neuroeconomics: Foundational issues and consumer relevance. En Haugvedt, C., Kardes, F., y Herr, P. (Eds.), Handbook of Consumer Psychology (pp. 1177-1214). Mahwah, NJ: Erbaum.

Fisher, C. E., Chin, L., \& Klitzman, R. (2010). Defining Neuromarketing: Practices and Professional Challenges. Harvard Review of Psychiatry, 18(4), 230-237. https://doi.org/10.3109/10673229.20 10.496623.

Fugate, D. L. (2008). Marketing services more effectively with neuromarketing research: a look into the future. Journal of Services Marketing, 22(2), 170-173. https://doi.org/10.1108/08876040810 862903.

Hazeldine, S. (2014). Neuro-sell: How neuroscience can power your sales success. UK: Kogan Page.

Henrich, J., Heine, S.J., \& Cnorezayan, A. (2010). The weirdest people in the world? Behavioral and Brain Sciences, 33, 61-135. https://doi.org/10.1017/S0140525X0999152X.

Hensel, D., lorga, A., Wolter, L., \& Znanewitz, J. (2017). Conducting neuromarketing studies ethicallypractitioner perspectives. Cogent Psychology, 4(1), 1320858. https://doi.org/10.1080/23311908 .2017 .1320858 .

Hilderbrand, M.L. (2016). Neuromarketing: An essential tool in the future of advertising and brand development. [Tesis doctoral, University of Texas].

Hsu, M. (2017). Neuromarketing: Inside the Mind of the Consumer. California Management Review, 59(4), 5-22. https://doi.org/10.1177/0008125617720208.

Hubert, M., \& Kenning, P. (2008). A current overview of consumer neuroscience. Journal of Consumer Behaviour, 7(4-5), 272-292. https://doi.org/10.1002/cb.251.

Illes, J., Kirschen, M.P., Edwards, E., Stanford, L.R., Bandettini, P., Cho, M.K., Ford, P.J., Glover, G.H., Kulynych, J., Macklin, R., Michael, D.B. \& Wolf, S.M. (2006). Ethics. Incidental findings in brain imaging research. Science, 311(5762), 783-784. https://doi.org/10.1126/science.1124665.

Karmarkar, U.R., \& Plassmann, H. (2019). Consumer Neuroscience: Past, Present, and Future. Organizational Research Methods, 22(1), 174-195. https://doi.org/10.1177/1094428117730598.

Kenning, P.H., \& Plassman, H. (2008). How neuroscience can inform consumer research. Neural Stytems and Rehabilitation Engineering, IEEE Transactions, 16(6), 532-538. https://doi.org/10.110 9/TNSRE.2008.2009788.

Knutson, B., \& Genevsky, A. (2018). Neuroforecasting Aggregate Choice. Current Directions in Psychological Science, 27(2), 110-115. https://doi.org/10.1177/0963721417737877.

Krampe, C., Strelow, E., Haas, A., \& Kenning, P. (2018). The application of mobile fNIRS to "shopper neuroscience" - first insights from a merchandising communication study. European Journal of Marketing, 52(1), 244-259. https://doi.org/10.1108/EJM-12-2016-0727.

Lee, N., Chamberlain, L., \& Brandes, L. (2018). Welcome to the jungle! The neuromarketing literature through the eyes of a newcomer. European Journal of Marketing, 52(1-2), 4-38. https://doi.org /10.1108/EJM-02-2017-0122.

Lee, N., Broderick, A.J., \& Chamberlain, L. (2007). What is neuromarketing? A discussion and agenda for future research. International Journal of Psychophysiology, 63(2), 199-204. https://doi.org/1 0.1016/j.jpsycho.2006.03.007.

Levy, M., \& Weitz, B.A. (2009). Retail Management. New York: MacGraw-Hill. 
Lewis, D. \& Bridger, D. (2005). Market researchers make increasing use of Brain imaging. https://bit.l y/3gu5510

Lim, W.M. (2018). Demystifying Neuromarketing. Journal of Business Research, 91, 205-220. https:// doi.org/10.1016/j.jbusres.2018.05.036.

Lin, M., Cross, S., Jones, W., \& Childers, T. L. (2018). Applying EEG in consumer neuroscience. European Journal of Marketing, 52(1), 66-91. https://doi.org/10.1108/EJM-12-2016-0805.

Lindstrom, M. (2008). Buyology: truth and lies about why we buy. Australia: Random House.

Lindstrom, M. (2010). Buyology. New York: Crown Publishing Group.

Marichamy, K., \& Sathiyavathi, J.K. (2014). Neuromarketing: The new science of consumer behavior. Tacftful Management Journal, 2(6).

McClure, S. M., Li, J., Tomlin, D., Cypert, K. S., Montague, L. M., \& Montague, P. R. (2004). Neural Correlates of Behavioral Preference for Culturally Familiar Drinks. Neuron, 44(2), 379-387. htt ps://doi.org/10.1016/j.neuron.2004.09.019.

Monge Benito, S., \& Fernández Guerra, V. (2012). Neuromarketing: Tecnologías, Mercado y Retos. Pensar La Publicidad. Revista Internacional De Investigaciones Publicitarias, 5(2), 19-42. https://d oi.org/10.5209/rev_PEPU.2011.v5.n2.37862.

Mucha, T. (2005). This is your brain on advertising. Business, 6(7), 35-37.

Murphy, E.R., Illes, J., \& Reiner, P.B. (2008). Neuroethics of neuromarketing. Journal of consumer behavior, 7(4), 293-302. https://doi.org/10.1002/cb.252.

Nave, G., Nadler, A., Dubois, D., Zava, D., Camerer, C., \& Plassmann, H. (2018). Singledose testosterone administration increases men's preference for status goods. Nature Communications, 9, 2433. https://doi.org/10.1038/s41467-018-04923-0.

Ohme, R., Reykowska, D., Weiner, D., \& Choromansk, A. (2009). Analysis of neurophysiological reactions to advertising stimuli by means of EEG and Galvanic Skin Response measures. Journal of Neuroscience, Psychology and Economics, 2(1), 21-31. https://doi.org/10.1037/a0015462.

Oliver, L. (2016). From exposure to purchase-Understanding the interaction of affect and cognition in consumer decision making. [Tesis doctoral, Hanken School of Economics].

Orzan, G., Zara, I.A., \& Purcarea, V.L. (2012). Neuromarketing techniques in pharmaceutical drugs advertising- A discussion and agenda for future research. Journal of Medicine and Life, 5(1), 428-432.

Perrachione, T.K., \& Perrachione, J.R. (2008). Brains and Brands: Developing mutually informative research in neuroscience and marketing. Journal of Consumer Behavior, 7(4), 303-318. https:// doi.org/10.1002/cb.253.

Plassmann, H., Ramsøy, T. Z., \& Milosavljevic, M. (2012). Branding the brain: A critical review and outlook. Journal of Consumer Psychology, 22(1), 18-36. https://doi.org/10.1016/j.jcps.2011.11.0 10.

Plassmann, H., Venkatraman, V., Huettel, S., \& Yoon, C. (2015). Consumer Neuroscience: Applications, Challenges, and Possible Solutions. Journal of Marketing Research, 52(4), 427-435. https://doi.org/10.1509/jmr.14.0048.

Plassmann, H., \& Weber, B. (2015). Individual Differences in Marketing Placebo Effects: Evidence from Brain Imaging and Behavioral Experiments. Journal of Marketing Research, 52, 493-510. $\underline{\mathrm{h}}$ ttps://doi.org/10.1509/jmr.13.0613.

Pop, C., Radomir, L., Ioana, M.A., \& Maria, Z.M. (2009). Neuromarketing - Getting Inside The Customer'S Mind. Annals of Faculty of Economics, 4, 804-807. 
Pop, A.N., Dabija, D.C., \& lorga, A.M (2014). Ethical responsibilities of neuromarketing companies in harnessing the market research - A global exploratory approach. AmfiteatruEconomic, XVI(35), 26-40.

Ramsoy, T.Z. (2014). Introduction to Neuromarketing and Consumer Science. Dinamarca: Neurons Inc. Riccio, A., Holz, E. M., Aricò, P., Leotta, F., Aloise, F., Desideri, L., Rimondini, M., Kübler, A., Mattia, D., \& Cincotti, F. (2015). Hybrid P300-based brain-computer interface to improve usability for people with severe motor disability: Electromyographic signals for error correction during a spelling task. Archives of Physical Medicine and Rehabilitation, 96(3), S54-S61. https://doi.org/1 0.1016/j.apmr.2014.05.029.

Rodrigues, F. (2011). Influencia do Neuromarketing nos processos de tomada de decisao. Viseu: Psicosma.

Roebuck, K. (2011). Neuromarketing: High-Impact strategies-What you need to know: Definitions, Adoptions, Impact, Benefits, Maturity, Vendors. Brisbane: Emereo Publishing.

Samuel, B.S., \& Pransanth, V.T. (2012). Neuromarketing: Is Campbell in Soup? IUP Journal of Marketing Management, 11(2), 76-100.

Sands, S. (octubre 2009). Sample Size Analysis for Brainwave Collection (EEG) Methodologies. https:// bit.ly/3zb3zJx.

Shiv, B., \& Fedorikhin, A. (1999). Heart and Mind in Conflict: The interplay of affect and cognition in consumer decision making. The Journal of Consumer Research, 26(3), 278-292. https://doi.o rg/10.1086/209563.

Sinek, S. (2009). Start with Why: How great leaders inspire everyone to take action. USA: Penguin Group.

Smidts, A. (october 25 de 2002). Kijkenin het brein: Over de mogelijkheden van neuromarketing. http ://hdl.handle.net/1765/308.

Solnais, C., Andreu-Perez, J., Sánchez-Fernández, J., \& Andréu-Abela, J. (2013). The contribution of neuroscience to consumer research: A conceptual framework and empirical review. Journal of Economic Psychology, 36, 68-81. https://doi.org/10.1016/j.joep.2013.02.011.

Stanton, S. J., Sinnott-Armstrong, W., \& Huettel, S. A. (2017). Neuromarketing: Ethical Implications of its Use and Potential Misuse. Journal of Business Ethics, 144(4), 799-811. https://doi.org/10. 1007/s10551-016-3059-0.

Thompson, D. (16 de enero de 2013). The irrational Consumer: Why Economics is dead wrong about how we make choices. https://bit.ly/2Smu91D.

Tovino, S.A. (2005). The confidentiality and privacy implications of functional magnetic resonance imaging. The Journal of Law, Medicine \& Ethics, 33, 844-850. https://doi.org/10.1111/j.1748-72 0X.2005.tb00550.x.

Yoon, C., Gonzales, R., Bechara, A., Berns, G., Dagher, A., Dubé, L., Huettel, S., Kable, J., Liberzon, I., Plassmann, H., Smidts, A., \& Spence, C. (2012). Decision neuroscience and consumer decisionmaking. Marketing Letters, 23(2), 473-485. https://doi.org/10.1007/s11002-012-9188-z.

Zajonc, R. (1980). Feeling and Thinking: Preferences need no inferences. American Psychologist, 35(2), 151-175. https://doi.org/10.1037/0003-066X.35.2.151. 